\title{
Comparative studies on the regulatory effects of raw and charred hawthorn on functional dyspepsia and intestinal flora
}

\author{
Zhiqiang Wei, Li Ai, Xi Chen, Lin Li, Li Wang, Wenxiang Fan, Xuekai Tang, \\ Fang Liang, Chunjie $\mathbf{W u}^{*}$ \\ College of Pharmacy, Chengdu University of Traditional Chinese Medicine, No. 1166 Liutai Avenue, Chengdu 610075, PR \\ China
}

*For correspondence: Email: wucjcdtcm@163.com; Tel: +86-028-61801001

\begin{abstract}
Purpose: To compare the effects of raw hawthorn $(\mathrm{RH})$ and charred hawthorn $(\mathrm{CH})$ on functional dyspepsia (FD) and intestinal flora (IF).

Methods: A rat model of FD was established through use of a chronic stimulator. Rat models were evaluated by the rat's physical state, body weight, diet, and histopathological examination. After RH or $\mathrm{CH}$ administration, the digestive function of the rats was evaluated by determining gastric emptying and intestinal propulsion rate, diversity of intestinal flora.

Results: $\mathrm{RH}$ and $\mathrm{CH}$ both improved gastric emptying and intestinal propulsion rate in FD group when compared to control group $(p<0.05)$. $\mathrm{CH}$ yielded higher treatment effectiveness than $\mathrm{RH}$. Sixteen phyla of microbiomes were recognized from all samples. After FD model establishment, the relative abundance of Lactobacillus, Lachnospiraceae and Bacteroidales decreased compared to normal control rats. On the other hand, the relative abundance of Helicobacter and Bacteroides in the model control group increased compared to normal control. After $\mathrm{RH}$ and $\mathrm{CH}$ treatment, the relative abundance of all dysregulated phyla was restored to varying degrees, but the levels after $\mathrm{CH}$ treatment were similar to those of the normal control group.

Conclusion: The relative abundance of intestinal flora of $F D$ model rats is significantly different from that of rats in normal control group. Thus, $\mathrm{RH}$ and $\mathrm{CH}$ intervention improves digestive function, and the mechanisms may be related to adjustment of gut dysbacteriosis.
\end{abstract}

Keywords: Raw Hawthorn, Charred Hawthorn, Functional dyspepsia, Intestinal flora,

\begin{abstract}
This is an Open Access article that uses a funding model which does not charge readers or their institutions for access and distributed under the terms of the Creative Commons Attribution License (http://creativecommons.org/licenses/by/4.0) and the Budapest Open Access Initiative (http://www.budapestopenaccessinitiative.org/read), which permit unrestricted use, distribution, and reproduction in any medium, provided the original work is properly credited.

Tropical Journal of Pharmaceutical Research is indexed by Science Citation Index (SciSearch), Scopus, International Pharmaceutical Abstract, Chemical Abstracts, Embase, Index Copernicus, EBSCO, African Index Medicus, JournalSeek, Journal Citation Reports/Science Edition, Directory of Open Access Journals (DOAJ), African Journal Online, Bioline International, Open-J-Gate and Pharmacy Abstracts
\end{abstract}

\section{INTRODUCTION}

Functional dyspepsia (FD) is one of the most prevalent functional diseases of the digestive tract, and the incidence of FD is increasing with each passing year. The global prevalence of uninvestigated dyspepsia in adults is $20.8 \%$ [1]. FD is characterized by an extended course, tending towards recrudescence of symptoms that include epigastric pain and burning, feeling bloated after a meal, early satiation, distension in the epigastric region, and nausea and vomiting in 
the absence of obvious organic pathologic changes [2]. All of these symptoms impact the quality of patient life. The precise mechanism of FD is not fully understood, and many current treatments are therapeutically unsatisfactory; hence, there is a need to develop a more effective treatment program.

Hawthorn refers to the ripe fruit of Crataegus pinnatifida Bge. var. major N.E.Br. or Crataegus pinnatifida Bge, belonging to the Rosaceae family of plants. In addition to being consumed as a food, hawthorn is a traditional Chinese herbal medicine that has been used for over a thousand years to treat dyspepsia, cardiodynia, hernia, and postpartum blood stasis. A series of investigations have demonstrated that hawthorn has numerous pharmacological effects on the digestive system [3]. Processing (pao zhi) is an integral step before clinical application of Traditional Chinese Medicine (TCM). In clinical practice, hawthorn is always charred for the treatment of dyspepsia. Some studies have also shown that $\mathrm{CH}$ is more effective in promoting gastric emptying and small intestine propelling in normal mice and atropine-loaded mice [4]. However, the mechanism behind the efficacy of hawthorn it cannot, as yet, be fully explained by any theory.

This paper studied the differences between the effects of $\mathrm{RH}$ and $\mathrm{CH}$ on the digestive system and intestinal flora of FD rats. In addition, this paper explores for the first time the possible mechanism by which of $\mathrm{CH}$ improves the digestive function.

\section{EXPERIMENTAL}

\section{Materials and reagents}

$\mathrm{RH}$ was collected from the Chengdu Hehuachi Chinese Herbal Medicine Market, which was identified by ChunJie Wu (College of Pharmacy, Chengdu University of Traditional Medicine) as being the dried fruit of Crataegus pinnatifida Bunge.

\section{Drug preparation}

To prepare the $\mathrm{CH}$, the empty heating pot was preheated to $250{ }^{\circ} \mathrm{C}$, and the $\mathrm{RH}$ was then added into the pot. The $\mathrm{RH}$ was stirred continuously even cooking until the surface of was brown.

Traditionally, $\mathrm{RH}$ and $\mathrm{CH}$ are boiled to treat dyspepsia. In this study, $50 \mathrm{~g}$ of $\mathrm{CH}$ or $\mathrm{RH}$ was weighed and extracted with boiling water twice for $1.5 \mathrm{~h}$. The resultant filtrate was concentrated to $150 \mathrm{ml}$ at $60^{\circ} \mathrm{C}$.

\section{Establishment of FD rat model}

The experimental program was approved by the Animal Care and Use Committee of Chengdu University of Traditional Chinese Medicine (No.20180209) and followed the international guidelines for animal studies [5] to minimize the pain and discomforts of the animals. Before experiments, rats were kept for 7 days at a temperature of $23 \pm 2{ }^{\circ} \mathrm{C}$ and a relative humidity of $55 \pm 2 \%$ on a $12 \mathrm{~h}$ light-dark cycle with a standard aseptic diet and distilled water ad libitum. Eighty male Sprague-Dawley (SD) rats (7 weeks of age) of specific pathogen free (SPF) clean grade weighing $180-220 \mathrm{~g}$ were supplied by the Chengdu Dashuo Experimental Animal Co., Ltd (Chengdu, China). Eighty rats were divided into a normal control group (NC, $n=20$ ) and a stress group (SG, $n=60$ ) randomly. Rats within the SG group were clamped $2 \mathrm{~cm}$ from the base of their tail for 30 min per day to make them angry, nervous and depressed [6]. The food intake was recorded of every day and the body weight every 3 days. In addition, the behavioral characteristics of the rats were observed for 21 days.

\section{Histopathological examination}

After 21 days of chronic stimulation, 6 rats in NC and 18 rats in the SG were randomly selected and sacrificed by vertebral dislocation. Stomach and duodenum tissues were collected immediately. The samples were opened to observe whether the tissues were whether normal and smooth, congested, edematous or eroded. The stomach and duodenum were subjected to histological examination after fixing with $4 \%$ paraformaldehyde and embedding in paraffin.

Pathological slices of rat stomach and duodenum were examined under an Olympus microscope (Olympus, Tokyo, Japan) after H\&E staining to determine morphological features [7].

\section{Gastric emptying and intestinal propulsion}

The rest of the rats in the SG were divided equally into three groups: the model control group (MG), the $\mathrm{RH}$ group (RG), and the $\mathrm{CH}$ group (CG). The $\mathrm{RG}$ and $\mathrm{CG}$ were given $\mathrm{RH}$ and $\mathrm{CH}$, respectively, for 7days, at a dose of $3 \mathrm{~g} / \mathrm{kg}$ per day. Gastric emptying and intestinal propulsion rates were measured to evaluate the digestive function according to a published method, with some modifications [8]. Briefly, on the seventh day, 6 rats were randomly selected from every group and fasted for 24 hours. One hour after the last $\mathrm{RH}$ or $\mathrm{CH}$ administration, all 
rats were given $2 \mathrm{~g}\left(\mathrm{Q}_{0}\right)$ of a semisolid paste (3.2 g starch, 3.2 g glucose, 4 g CMC-Na, 100 mL distillated water, $0.14 \mathrm{~mL}$ pure black ink). Twenty minutes later, the rats were sacrificed by cervical dislocation. The stomach (from cardia to pylorus) was exposed and removed by laparotomy to measure the stomach weight $\left(Q_{1}\right)$ and stomach net weight $\left(Q_{2}\right)$ and determine the gastric residual rate (GR) according to the formula 1 . The small intestine (from the pylorus to the ileocecal) was then removed to separate the mesentery and placed on filter paper and straightened gently to measure the distance of ink advancement $\left(L_{1}\right)$ and intestinal length $\left(L_{2}\right)$. The intestinal propulsion rate (IR) for each rat was calculated according to the formula 2 .

$$
\begin{aligned}
& \mathrm{GR}(\%)=\frac{Q 1-Q 2}{Q 0} \times 100 \ldots \ldots \ldots \ldots(1) \\
& \mathrm{IR}(\%)=\frac{21}{22} \times 100 \ldots \ldots \ldots \ldots(2) \\
& \text { Polymerase chain reaction (PCR) } \\
& \text { amplification and sequencing }
\end{aligned}
$$

\section{Polymerase chain reaction (PCR) amplification and sequencing}

Stool samples were obtained from the colon of the remaining rats under sterile conditions. To amplify the V3-V4 region of the 16S rRNA gene for Illumina deep sequencing, universal primers, 338 F: 5'-ACTCCTACGGGAGGCAGCA-3' and 806 R: 5'-GGACTACHVGGGTWTCTAAT-3', were used. The PCR was performed in a total reaction volume of $20 \mu \mathrm{L}: 13.25 \mu \mathrm{L} \mathrm{H} \mathrm{H}_{2} \mathrm{O}, 2.0 \mu \mathrm{L} 10$ $\times$ PCR ExTaq Buffer, $0.5 \mu \mathrm{L}$ DNA template (100 $\mathrm{ng} / \mathrm{mL}), 1.0 \mu \mathrm{L}$ prime 1 (10 $\mathrm{mmol} / \mathrm{L}), 1.0 \mu \mathrm{L}$ prime $2(10 \mathrm{mmol} / \mathrm{L}), 0.25 \mu \mathrm{L}$ dNTP $2.0 \mu \mathrm{L}$, ExTaq $(5 \mathrm{U} / \mathrm{mL})$. After an initial denaturation at $95^{\circ} \mathrm{C}$ for $5 \mathrm{~min}$, amplification was performed by 30 cycles of incubations for $30 \mathrm{sec}$ at $95^{\circ} \mathrm{C}, 20 \mathrm{sec}$ at 58 ${ }^{\circ} \mathrm{C}$, and $6 \mathrm{sec}$ at $72{ }^{\circ} \mathrm{C}$, followed by a final extension at $72{ }^{\circ} \mathrm{C}$ for $7 \mathrm{~min}$. Then the amplified products were purified and recovered using a $1.0 \%$ agarose gel electrophoresis method. The library construction and sequencing steps were performed by Beijing Biomarker Technologies Co. Ltd (Beijing, China).

\section{Bioinformatic analysis}

The bioinformatic analysis in this study was completed on the Biomarker biocloud platform (www.biocloud.org). To obtain the raw tags, paired-end reads were merged by FLASH (v1.2.7, http://ccb.jhu.edu/software/FLASH/) (Magoč, 2011). Raw tags were then filtered and clustered in the next steps. The merged tags were compared to the primers, and the tags with more than six mismatches were discarded by the FASTX-Toolkit. Tags with an average quality score $<20$ in a 50 bp sliding window were truncated using Trimmomatic (available from http://www.usadellab.org/cms/?page=trimmomati c) (Bolger, 2014) and tags shorter than $350 \mathrm{bp}$ were removed. We identified possible chimeras by employing UCHIME, a tool included in mothur (http://drive5.com/uchime).

The denoised sequences were clustered using USEARCH (version 10.0) and tags with similarity $\geq 97 \%$ were regarded as operational taxonomic units (OTUs). Taxonomy was assigned to all OTUs by searching against the Silva databases (Release128, http://www.arb-silva.de.) using the uclust within QIIME.

\section{Statistical analysis}

Data are presented as mean \pm SD. Statistical comparisons were made by one-way analysis of variance (ANOVA) using SPSS software (version 18.0, USA), followed by Dunnet $t$ multiple comparison test. A P value of less than 0.05 was considered as the significance level.

\section{RESULTS}

\section{Validation data for FD model}

An FD model in rats was developed through of a chronic stimulator as described in as described in the Experimental section After 21 days rats in the SG presented with losses in body weight, dull and messy hair, slow reaction times, sluggish spirits, pasty and loose stools, poor appetites, and lassitude. These signs are similar to the symptoms of FD that are observed in humans. Compared with the NC, the body weight and food intake of SG rats were obviously decreased (Table 1), indicating that the SG rats show the symptoms of dyspepsia.

Table 1: Body weight and food intake of rats

\begin{tabular}{lccccccc}
\hline Group & $\mathbf{0}$ & $\mathbf{3}$ & $\mathbf{6}$ & $\mathbf{9}$ & $\mathbf{1 2}$ & $\mathbf{1 5}$ & $\mathbf{1 8}$ \\
\hline Body weight (mg) & & & & & & & \\
NC & 207.5 & 219.5 & 239.6 & 257.4 & 271.3 & 284.2 & 299.5 \\
SG & 209.3 & 216.0 & 228.7 & 237.6 & 243.1 & 247.5 & 251.8 \\
Food intake (mg) & & & & & & & \\
NC & 22.1 & 22.5 & 22.8 & 23.2 & 23.4 & 23.9 & 24.3 \\
SG & 22.3 & 21.7 & 21.2 & 20.7 & 20.2 & 19.8 & 19.6 \\
\hline
\end{tabular}




\section{Histological features}

The tissue structure of the stomach and duodenum of SG was morphologically normal with distinct structural layers and regular structural arrangements. Duodenal villi were neatly arranged and structurally intact. Mucosal epithelial cells arranged densely, and the morphology of the cells was regular. There was no fibrous hyperplasia in the submucosal connective tissue, vasodilation or inflammatory cell infiltration (Figure 1). The results show that the process of model establishment did not cause organic diseases and that the FD rat model was developed successfully.

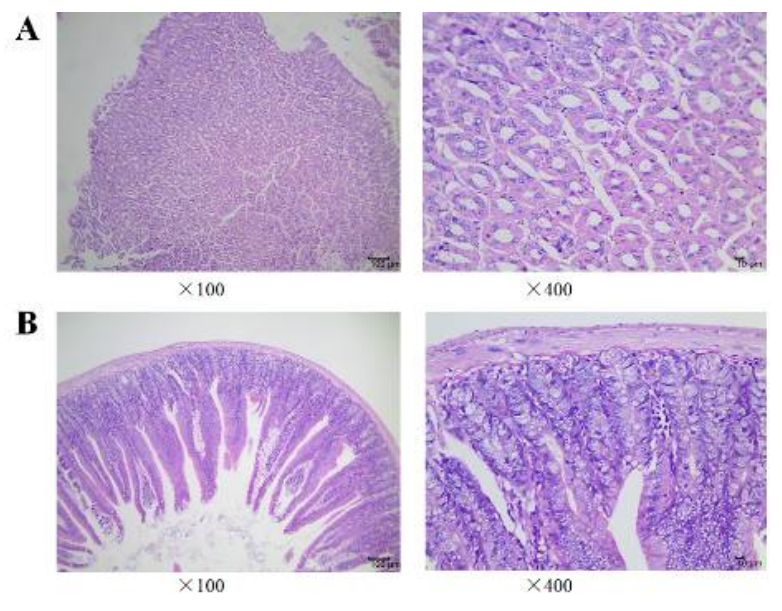

Figure 1: Stomach (A) and duodenum (B) sections of FD rats

\section{Effect of RH and $\mathrm{CH}$ on gastric emptying and intestinal propulsion}

Compared to NC, rats in MG showed a significant increase of semisolid paste residue in the stomach $(p<0.05)$, and a significant decrease of intestinal propelling $(p<0.01)$. $\mathrm{RH}$ and $\mathrm{CH}$ both significantly enhanced gastric emptying and intestinal propulsion $(p<0.05)$, although $\mathrm{CH}$ was more effective than $\mathrm{RH}$ (Figure 2).
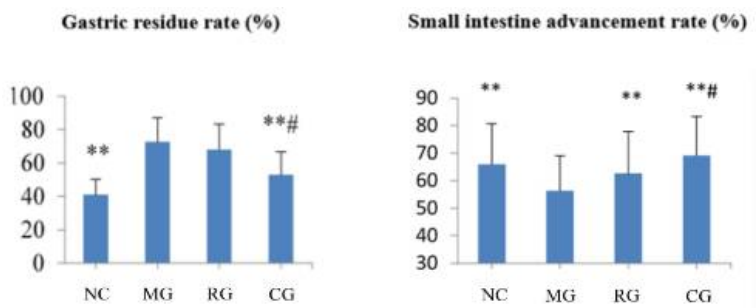

Figure 2: Gastric residue rate and intestinal propulsion rate. Data are presented as mean $\pm \mathrm{SD} ;{ }^{* *} p$ $<0.01$ vs. the FD model rats; $\# p<0.05$ vs. $\mathrm{RH}$-treated rats

\section{Effect of RH and $\mathrm{CH}$ on intestinal flora of FD rats}

A total of 16 phylum and 159 genera of microbiomes were found in all rat stool samples (Figure 4). The microbiome composition for all groups was similar at the phyla level. The abundance of probiotics, Lactobacillus, Lachnospiraceae, and Bacteroidales was higher in the NC rats relative to the MG rats. On the contrary, the abundance of Helicobacter and Bacteroides was higher in the FD MG relative to the NC. After both $\mathrm{RH}$ and $\mathrm{CH}$ treatment, the relative abundance of Lactobacillus and Bacteroidales was significantly increased, although the degree of relative abundance growth was greater for $\mathrm{CH}$. At the same time, the relative abundance of Helicobacter and Bacteroides in both $C G$ and $R G$ was significantly decreased, although the decrease was greater for $C G$ and normal levels were reached (Figure 3).

\section{DISCUSSION}

FD is defined by Rome III Committees [9] as a chronic and recurring disease without any organic lesions, which mainly manifests as a sensation of pain or burning in the epigastrium, early satiety, fullness during or after a meal, vomiting, or a combination of the latter symptoms. Increasing evidence suggests that psychosocial factors and psychiatric disorders, especially anxiety and depression, are associated with FD [10]. Therefore, a rat stress stimulation model was chosen for use in this study. Psychological and physiological stresses, such as clamping the tails, have been shown to inhibit antral motility and gastric emptying in animals. This principal has been widely used in establishing a model for FD [11]. Stress stimulation, without any medical intervention, was the most relevant model of FD pathogenesis with simulation of a pathological state that includes tension and, to some extent, patient anxiety.

In this experiment, the rat FD model was validated by recording food and water intake, measuring changes in body weight, observing the rats' ethologic characteristics, testing gastric emptying and intestinal propulsion, and conducting histological examinations. Compared to the rats in the NC, the model rats showed several FD symptoms, such as dull and messy hair, slow reaction times, sluggish spirits, pasty and loose stools, bad appetites, lassitude, decreased food intake, and slower increases in body weight. Histological examination showed no organic lesions. All of these results showed that 

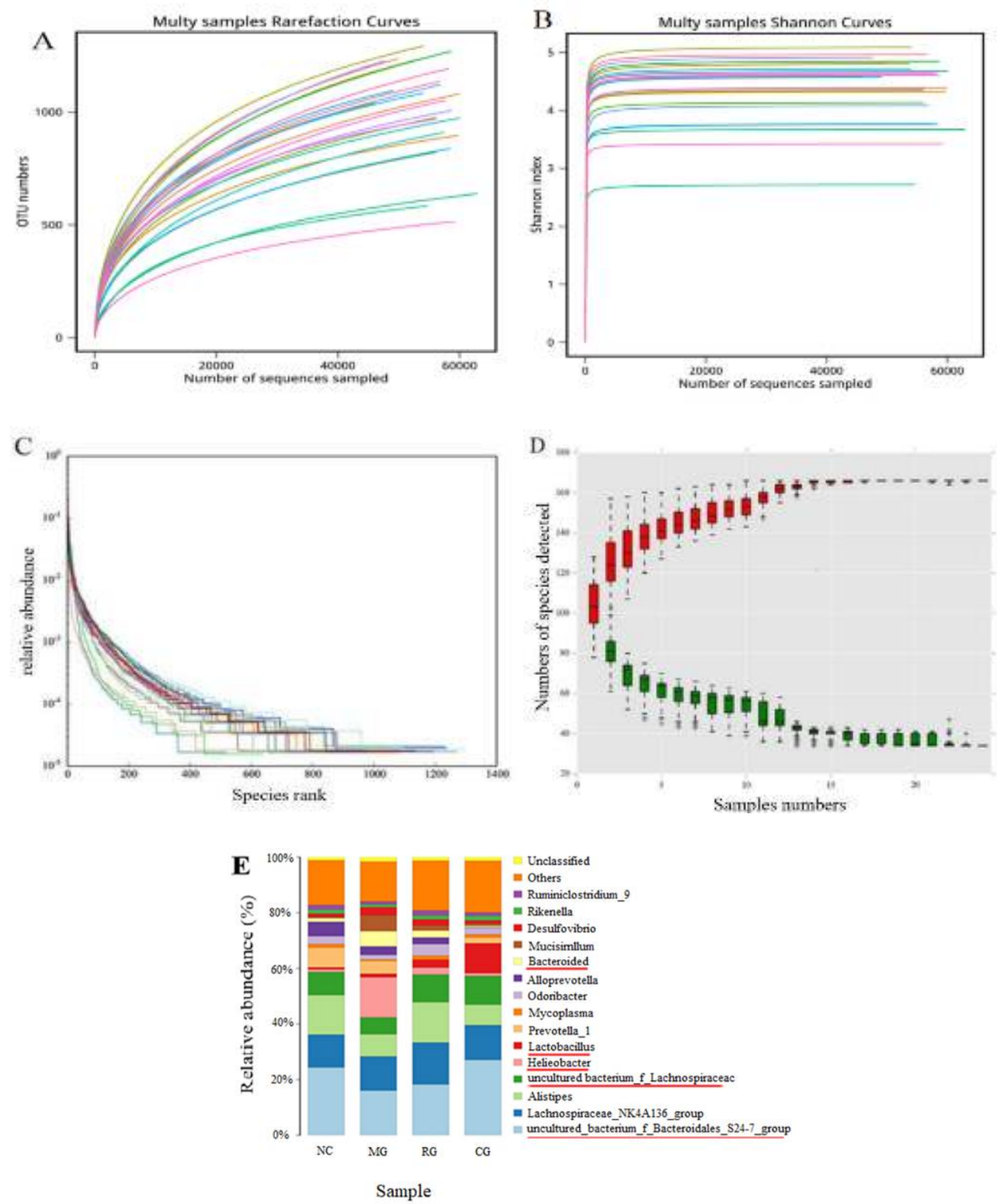

Figure 3: Effects of $\mathrm{RH}$ and $\mathrm{CH}$ on the intestinal flora of FD rats. (A) Rarefaction Curve; (B) Shannon Index; (C) Rank Abundance Curve; (D) Species Detected Accumulation Curve; (E) intestinal flora species distribution

we successfully established an FD model. $\mathrm{CH}$ and $\mathrm{RH}$ were shown to promote digestion, as indicated by the gastric residue rate and intestinal propulsion rate tests.

In recent years, many researchers have shown that the intestinal flora is related to our health. When the balance of intestinal flora is broken, the host may develop an illness, such as type 2 diabetes, irritable bowel syndrome (IBS), and FD $[12,13]$. Research has shown that some traditional Chinese herbal medicine may act by adjusting the composition or equilibrium of the intestinal flora [14]. Therefore, we tried to reveal the mechanism of $\mathrm{RH}$ and $\mathrm{CH}$ in treating FD from the perspective of intestinal flora. Bacteroidetes is one of the predominant microflora in the human gut. Many Bacteroidetes are known to participate in the metabolic processing of polysaccharides, steroids, and bile acids. In addition, a decrease in the relative abundance of Bacteroidetes is suggested to be related to obesity, IBS, diabetes, and other conditions $[15,16]$. Many researchers have reported that Lactobacillus has a number of health benefits, such as anti-pathogenic, immunostimulatory, and catabolic activities [17,18]. Most importantly, some research showed that Lactobacillus can regulate and control emotion and anxiety of mice across the brain-gut axis [19]. Members of the Lachnospiraceae family have been shown to be associated with the production of butyric acid, a substance that is important for both microbial and host epithelial cell growth [20]. In addition, the 
increases of relative abundance of harmful bacteria, such as Helicobacter and Bacteroide are likely associated with celiac disease [21], FD, gastritis, peptic ulcer disease, and even gastric cancer $[22,23]$.

\section{CONCLUSION}

The findings of this study demonstrate that the relative abundance of the intestinal flora in FD rats established by stress stimulation is significantly different from that found in rats within NC. The results indicate that $\mathrm{RH}$ and $\mathrm{CH}$ interventions are effective in the treatment of $\mathrm{FD}$, and that $\mathrm{CH}$ is a more effective than $\mathrm{RH}$. This may be due to the fact that $\mathrm{CH}$ is more effective in adjusting gut dysbacteriosis. This study therefore provides a new train of thought on the strategy to treat FD. However, further in vitro and in vivo studies are required in this regard.

\section{DECLARATIONS}

\section{Acknowledgement}

This study was financially supported by the National Natural Science Foundation of China (no. 81403105).

\section{Conflict of interest}

No conflict of interest is associated with this work.

\section{Contribution of authors}

The authors declare that this work was done by the authors named in this article and all liabilities pertaining to claims relating to the content of this article will be borne by them. C.-J. Wu conceived the research. Z, -Q. Wei and X. Chen drafted the manuscript. All authors have read and approved the manuscript for publication.

\section{REFERENCES}

1. Ford AC, Marwaha A, Sood R, Moayyedi P. Global prevalence of, and risk factors for, uninvestigated dyspepsia: a meta-analysis. Gut 2015; 64(7): 10491057.

2. Camilleri M. Functional Dyspepsia and Gastroparesis. Digest Dis 2016; 34(5): 491.

3. Huang SS, Lin Y, Diao YP, Liu ZH, Zeng YH, Zhang $H L$. Effects of charred Fructus crataegi alcohol extract on contractility of isolated rat gastric and intestine muscle strips. Progr Mod Biomed 2009; 9(4): 612-614.

4. Zhang SY, Sun GX, Feng B. Study on the influence of different processed products of hawthorn on gastrointestinal motility. Yunnan J Trad Chin Med Mater Med 2010; 31(5): 60-62.

5. Association AMPSA. Guidelines for ethical conduct in the care and use of animals. Journal of the Experimental Analysis of Behavior. 2013; 45(2):127-132.

6. Guo Y, Yang JQ, Li YJ, Song YL, Wei W. Overview of the functional dyspepsia animal models in China. World $J$ Integr Trad West Med; 2015(8): 1182-1184.

7. Peng $W$, Qiu $X Q$, Shu $Z H$, Liu QC, Hu MB, Han $T$, Rahman K, Qin L, Zheng CJ. Hepatoprotective activity of total iridoid glycosides isolated from Paederia scandens (Lour.) Merr. var. Tomentosa. J Ethnopharmacol 2015; 174: 317-321.

8. Sun JB, Zhang HL, Qin Y, Fan RY, Huang QW. Effects of Banxiaqu on the gastrointestinal motility in mice. Asia Pacific Trad Med 2018; 14(5): 13-16.

9. Talley NJ, Ford AC. Functional Dyspepsia. Drugs 1993; 45(6): 918-930.

10. Van OL, Aziz Q. The role of psychosocial factors and psychiatric disorders in functional dyspepsia. Nature Rev Gastroenterol Hepatol 2013; 10(3): 158-167.

11. Wei W, Li X, Hao J, Zhang R, Guo J, Zong Y, Lu Y, Qu S, Tian J. Proteomic analysis of functional dyspepsia in stressed rats treated with traditional Chinese medicine "Wei Kangning". J Gastroenterol Hepatol 2011; 26(9): 1425-1433.

12. Barczynska R, Bandurska K, Slizewska K, Litwin M, Szalecki M, Libudzisz Z, Kapusniak J. Intestinal Microbiota, Obesity and Prebiotics. Polish Soc Microbiol 2015; 64(2): 93-100.

13. Barbara G, Feinle-Bisset C, Ghoshal UC, Quigley EM, Santos J, Vanner S, Vergnolle N, Zoetendal EG. The intestinal microenvironment and functional gastrointestinal disorders. Gastroenterol 2016; 150(6): 1305-1318.

14. Peng Y, Wang Z, Lu Y, Wu CF, Yang JY, Li XB. Intestinal microflora molecular markers of spleen-deficient rats and evaluation of traditional Chinese drugs. World $\mathrm{J}$ Gastroenterol 2009; 15(18): 2220-2227.

15. Rajilić-Stojanović $M$, Biagi E, Heilig $H G$, Kajander $K$, Kekkonen RA, Tims S, DeVos WM. Global and deep molecular analysis of microbiota signatures in fecal samples from patients with irritable bowel syndrom. Gastroenterol 2011; 141(5): 1792-1801.

16. Pamer EG. Resurrecting the intestinal microbiota to combat antibiotic-resistant pathogens. Science 2016; 352(6285): 535-538.

17. Gensollen T, lyer S S, Kasper D L, Blumberg RS. How colonization by microbiota in early life shapes the immune system. Science 2016; 352(6285): 539-544.

18. Wall $R$, Marques TM, O'Sullivan $O$, Ross RP, Shanahan $F$, Quigley EM, Dinan TG, Kiely B, Fitzgerald GF, et al. Contrasting effects of Bifidobacterium breve NCIMB 702258 and Bifidobacterium breve DPC 6330 on the composition of murine brain fatty acids and gut microbiota. Am J Clin Nutr 2012; 95(5): 1278-1287.

19. Liu WH, Chuang HL, Huang YT, Wu CC, Chou GT, Wang $S$, Tsai YC. Alteration of behavior and monoamine

Trop J Pharm Res, February 2019; 18(2): 338 
Wei et al

levels attributable to Lactobacillus plantarum, PS128 in germ-free mice. Behav Brain Res 2015; 298: 202-209.

20. Meehan CJ, Beiko RG. A phylogenomic view of ecological specialization in the Lachnospiraceae, a family of digestive tract-associated bacteria. Genome Biol Evol 2014; 6(3): 703-713.

21. Sánchez E, Laparra JM, Sanz Y. Discerning the role of Bacteroides fragilis in celiac disease pathogenesis. Appl Environ Microbiol 2012; 78(18): 6507.

22. Watari J, Chen N, Amenta PS, Fukui H, Oshima $T$, Tomita T, Miwa H, Lim KJ, Das KM. Helicobacter pylori associated chronic gastritis, clinical syndromes, precancerous lesions, and pathogenesis of gastric cancer development. World J Gastroenterol 2014; 20(18): 5461-5473.

23. Peng $W$, Shen $H$, Lin B, Han P, Li CH, Zhang $Q Y, Y e B Z$, Rahman K, Xin HL, Qin LP, Han T. Docking study and antiosteoporosis effects of a dibenzylbutane lignan isolated from Litsea cubeba targeting Cathepsin $K$ and MEK1. Med Chem Res 2018; 27(9): 2062-2070. 